\title{
Calorímetro diferencial para estudo do padrão de aquecimento gerado por feixes ultrassônicos terapêuticos
}

\author{
Thaís Pionório Omena*, Rejane Medeiros Costa, Wagner Coelho de Albuquerque Pereira, \\ Marco Antônio von Krüger
}

Resumo O presente trabalho descreve o projeto e construção de um calorímetro diferencial com a finalidade de avaliar o aquecimento gerado por feixes ultrassônicos em níveis terapêuticos. O calorímetro consiste em duas câmaras cilíndricas de alumínio idênticas (de medição e de referência), preenchidas com um material mimetizador de tecido biológico (phantom). Cada câmara possui seis termopares tipo E (diâmetro 0,24 mm), posicionados ao longo do eixo central de propagação da onda, entre as profundidades 10-60 mm, distantes $10 \mathrm{~mm}$ entre si. Foi levantada a curva de potência e estimado o valor da área de radiação efetiva (ERA) dos dois transdutores utilizados. A partir destes dados, a intensidade efetiva foi determinada. Para avaliar o calorímetro, foi utilizado um equipamento de ultrassom de Fisioterapia, operando nas frequências nominais 1 e $3 \mathrm{MHz}$, modo contínuo, intensidades nominais 0,$5 ; 1,0 ; 1,5 \mathrm{e} 2,0 \mathrm{~W} \cdot \mathrm{cm}^{-2}$ e tempo de irradiação 180 segundos. Uma sequência de oito protocolos de medição foi realizada dez vezes. Para ambas as frequências, houve um declínio do aquecimento ao longo da profundidade e a região do "phantom" que mais aqueceu foi a que corresponde à profundidade de $10 \mathrm{~mm}$, em todas as intensidades. O maior aquecimento ocorreu a $2,0 \mathrm{~W} \cdot \mathrm{cm}^{-2}$, com médias de $6,7 \pm 1,0^{\circ} \mathrm{C}$ e $12,6 \pm 1,2^{\circ} \mathrm{C}$, a $1 \mathrm{MHz}$ e $3 \mathrm{MHz}$, respectivamente. O calorímetro proposto mostrou-se útil na caracterização de feixes ultrassônicos aplicados em Fisioterapia, principalmente na identificação de possíveis máximos locais de temperatura (pontos quentes) que ocorrem ao longo do eixo principal do feixe.

Palavras-chave Calorímetro diferencial, Ultrassom, Aquecimento, Fisioterapia.

\section{Differential calorimeter for studying the heating pattern generated by therapeutic ultrasonic beams}

\begin{abstract}
The present work describes the project and construction of a differential calorimeter designed to evaluate the heating generated by ultrasound beam in therapeutic levels. The calorimeter consists of two identical aluminum cylindrical chambers (for measurement and reference) filled with biological tissue mimicking material (phantom). Each chamber is fitted with six thermocouples type E (0.24 $\mathrm{mm}$ diameter) disposed along the wave propagation central axis, between the depths of 10-60 $\mathrm{mm}$, distant $10 \mathrm{~mm}$ from each other. The power curve and the value of the effective radiation area (ERA) of the two transducers were estimated. From these data, the effective intensity was determined. The calorimeter was tested by using an ultrasound equipment of Physiotherapy, operating at the frequencies 1 and $3 \mathrm{MHz}$, continuous mode, nominal intensities $0.5,1.0,1.5$ and $2.0 \mathrm{~W} \cdot \mathrm{cm}^{-2}$ and irradiation time $180 \mathrm{~seconds}$. A sequence of eight protocols of measurement was repeated 10 times. At both frequencies, there was a decline of heating along the depth and the phantom region that heated the most corresponded to $10 \mathrm{~mm}$ of depth, in all intensities employed. The greatest increase in temperature occurred after application of $2.0 \mathrm{~W} \cdot \mathrm{cm}^{-2}$, with averages of $6.7 \pm 1.0^{\circ} \mathrm{C}$ and $12.6 \pm 1.2^{\circ} \mathrm{C}$, at $1 \mathrm{MHz}$ and $3 \mathrm{MHz}$, respectively. The proposed calorimeter may be useful for identifying possible local temperature maxima (hot spots) that appear along the central axis beam.
\end{abstract}

Keywords Differential calorimeter, Ultrasound, Heating, Physiotherapy. 


\section{Introdução}

Ondas ultrassônicas são utilizadas em Fisioterapia como um recurso para gerar o aquecimento, reduzir a inflamação e acelerar a cicatrização dos tecidos (Guirro e Guirro, 2004). O aquecimento dos tecidos ocorre devido à absorção do feixe ultrassônico, durante sua propagação (Fish, 1990).

Sabendo-se que dose é a combinação entre intensidade e tempo de irradiação, a dose ideal é descrita como aquela que aumenta a temperatura dos tecidos até a faixa de $40-45^{\circ} \mathrm{C}$, e a mantém por, no mínimo, 5 minutos (Guirro e Guirro, 2004; Prentice, 2004). Nesta faixa de temperatura, os tecidos são submetidos aos efeitos fisiológicos desejados, tais como aumento do fluxo sanguíneo, redução do espasmo muscular e da rigidez articular, diminuição da inflamação e aumento da extensibilidade das estruturas colágenas. Outros autores descrevem os efeitos terapêuticos, de acordo com o aumento da temperatura: acima de $1{ }^{\circ} \mathrm{C}$, há aumento do metabolismo e estímulo à cicatrização; de 2 a $3{ }^{\circ} \mathrm{C}$, há redução da dor e do espasmo muscular; e a partir de $4{ }^{\circ} \mathrm{C}$, há aumento da extensibilidade do tecido colágeno e redução da rigidez articular (Draper e Ricard, 1995; Hayes et al., 2004; Merrick et al., 2003; Prentice, 2004). Para gerar tais efeitos, é necessário aplicar uma dosagem ótima no tecido tratado, de acordo com a área, profundidade e tipo de lesão. Após a seleção da freqüência do transdutor, os parâmetros passíveis de serem manipulados no equipamento são: modo de irradiação, intensidade e o tempo.

A radiação ultrassônica em Fisioterapia submete os tecidos ao campo próximo, que é a região mais próxima à face do transdutor, caracterizada pela não-uniformidade do feixe em função dos máximos e mínimos de pressão devidos às interferências construtivas (em fase) e destrutivas (fora de fase) das ondas provenientes da região central e das bordas do transdutor. Esta região apresenta, portanto, diferentes níveis de intensidade de energia ultrassônica. Para evitar a formação de pontos-quentes (hot spots) e distribuir a energia ultrassônica mais uniformemente na área de tratamento, é recomendada a técnica de movimentação do transdutor. Apesar de fazer parte da conduta fisioterapêutica, ainda não existe um protocolo eficaz de padrão de movimentação do transdutor, porém alguns autores preconizam uma velocidade de movimentação de $4 \mathrm{~cm} \cdot \mathrm{s}^{-1}$ (Cameron, 2009; Prentice, 2004). Este é um fator importante a ser considerado, já que contribui para uma distribuição mais homogênea do aquecimento na área de tratamento. Já os fatores que estão relacionados ao resfriamento são: a perfusão sanguínea e a condução de calor para os tecidos adjacentes.
A terapêutica por ultrassom depende, basicamente, de dois fatores: o protocolo de aplicação adotado e o desempenho do equipamento. Quando os equipamentos de ultrassom são calibrados, são verificadas diferenças significativas entre os valores nominais (fornecidos pelo fabricante) e efetivos (estimados) para a área de radiação efetiva (ERA), potência e intensidade (Ishikawa, 2000; Johns et al., 2007, Straub et al., 2008).

Tal variabilidade contribui para a ausência ou redução dos benefícios pretendidos ou para o agravamento da lesão. A calibração de equipamentos de ultrassom fisioterapêutico tem importância fundamental no sucesso da reabilitação.

Uma das formas de se estudar o aquecimento por ultrassom é utilizar calorímetros, que são instrumentos de medição da quantidade de calor fornecida ou recebida por um corpo (Delchar e Melvin, 1994; Furtado, 2005; Ramos, 2004).

O objetivo deste trabalho foi projetar e construir um calorímetro diferencial para se estudar o aquecimento, gerado por ultrassom em níveis terapêuticos, ao longo da profundidade de um meio mimetizador de tecido biológico (também denominado phantom).

\section{Materiais e Métodos}

Este estudo consistiu em três etapas: (i) montagem do calorímetro; (ii) calibração do equipamento ultrassônico; e (iii) elaboração e testes dos protocolos de aquecimento.

i) Montagem do calorímetro: O calorímetro diferencial foi formado por dois tubos cilíndricos de alumínio, de dimensões idênticas (comprimento $110 \mathrm{~mm}$, diâmetro interno $45 \mathrm{~mm}$ ) contendo uma matriz linear de seis termopares do tipo E (constantan-cromel), de diâmetro $0,24 \mathrm{~mm}$. Os termopares foram confeccionados em laboratório. Cada termopar diferencial possuiu duas junções, colocadas nas porções centrais do tubo de medição e de referência. Os termopares ficaram dispostos em uma matriz ao longo do tubo nas profundidades de $10 \mathrm{a}$ $60 \mathrm{~mm}$, espaçados de $10 \mathrm{~mm}$ entre si, de forma que a temperatura detectada foi a diferença de uma junção em relação à de referência, à mesma profundidade, e suas extremidades foram conectadas a um equipamento para aquisição de sinais (Spider 8, HBM).

$\mathrm{O}$ phantom empregado era à base de Agar, homogêneo e com propriedades ultrassônicas e térmicas equivalentes às da gordura. Propriedades acústicas medidas com transdutor de $1 \mathrm{MHz}$ : coeficiente de atenuação $0,22 \pm 0,01 \mathrm{~dB} \cdot \mathrm{cm}^{-1}$ e velocidade de propagação de $1520 \pm 36 \mathrm{~m} \cdot \mathrm{s}^{-1}$. Propriedades térmicas: difusividade térmica $1,13 \pm 0,01 \mathrm{~mm}^{2} \cdot \mathrm{s}^{-1}$, condutividade térmica $0,53 \pm 0,03 \mathrm{~W} \cdot \mathrm{m}^{-1} \cdot{ }^{\circ} \mathrm{C}^{-1} \mathrm{e}$ calor específico $3477 \pm 204 \mathrm{~J} \cdot \mathrm{kg}^{-1} \cdot{ }^{\circ} \mathrm{C}^{-1}$ (Basto, 2006). 
ii) Calibração do equipamento ultrassônico: $A$ calibração do equipamento ultrassônico foi realizada no laboratório de Ultrasssom (COPPE/UFRJ) e consistiu na estimativa dos valores de intensidade efetiva. Para isso, foram realizados dois procedimentos: a) calibração da potência do aparelho pela balança de força de radiação (UPM-DT-1\&10, Ohmic Instruments, EUA) conforme a norma NBR/IEC 1689 (Associação..., 1998); e b) levantamento da ERA conforme a norma NBR/IEC 1689 (Associação..., 1998). A estimativa da ERA foi realizada pelo mapeamento do campo acústico por hidrofone (Precision Acoustics, LTD), movimentado por um sistema de varredura mecânico utilizando motores de passo e controlado por um microcomputador.

iii) Elaboração e testes dos protocolos de aquecimento: A montagem experimental para testar os protocolos de aquecimento consistiu de: calorímetro, equipamento de ultrassom fisioterapêutico (Avatar III, KLD), placa absorvedora, equipamento de aquisição de sinais (Spider 8) e microcomputador (Figura 1).

O transdutor ultrassônico foi fixado a $1 \mathrm{~cm}$ do phantom, imerso em água. Foi adotada aplicação estática do transdutor durante a irradiação.

O protocolo de irradiação constituiu na combinação de uma freqüência (1 ou $3 \mathrm{MHz}$ ) com uma intensidade nominal $\left(0,5 ; 1,0 ; 1,5\right.$ e $\left.2,0 \mathrm{~W} \cdot \mathrm{cm}^{-2}\right)$ aplicadas em modo contínuo, durante 180 segundos. Essas combinações geraram oito protocolos. Cada um deles foi aplicado dez vezes, totalizando 80 experimentos.

\section{Resultados}

Foi construído um calorímetro diferencial para testar protocolos de irradiação gerados por equipamentos de ultrassom fisioterapêuticos (Figura 2).

A partir da medição da potência ultrassônica na balança de força de radiação (UPM-DT-1\&10, Ohmic Instruments, EUA), foi realizada uma regressão linear entre os valores da potência nominal e medida, para verificar a linearidade do equipamento. Foram obtidas duas equações, a $1 \mathrm{MHz}(\mathrm{y}=1,05 \mathrm{x}-0,31)$ e a $3 \mathrm{MHz}$ $(y=0,99 x-0,28)$, onde " $x$ " representa a potência nominal e " $y$ " a potência aferida. Ambas apresentaram coeficiente de determinação $\left(\mathrm{R}^{2}\right)$ de 0,99 .

AERA foi estimada por um sistema de mapeamento computadorizado, no qual o hidrofone varre o campo acústico gerado pelo transdutor, no interior de um tanque com água, com base na norma NBR/IEC 1689 (Associação..., 1998). De forma simplificada, a ERA foi calculada a partir das quatro áreas de seç̧ão transversal do feixe (ASTF), definida como a menor área que engloba $75 \%$ da potência irradiada pelo transdutor. A varredura ao longo do eixo central do feixe determina a posição de transição entre os campos próximo e distante (Zn). Por fim, a ERA é estimada a partir da regressão linear sobre o valor das quatro ASTF multiplicada por um fator adimensional (Fac) (Alvarenga et al., 2001). Os materiais utilizados foram: tanque de dimensões $(90 \mathrm{~cm} \times 60 \mathrm{~cm} \times 50 \mathrm{~cm}$ ), motor de passo que opera em três eixos em passos de 0,174 mm, gerador de sinais ( $A F G 3021$, Tektronix), osciloscópio (TDS3014B, Tektronix), transdutor
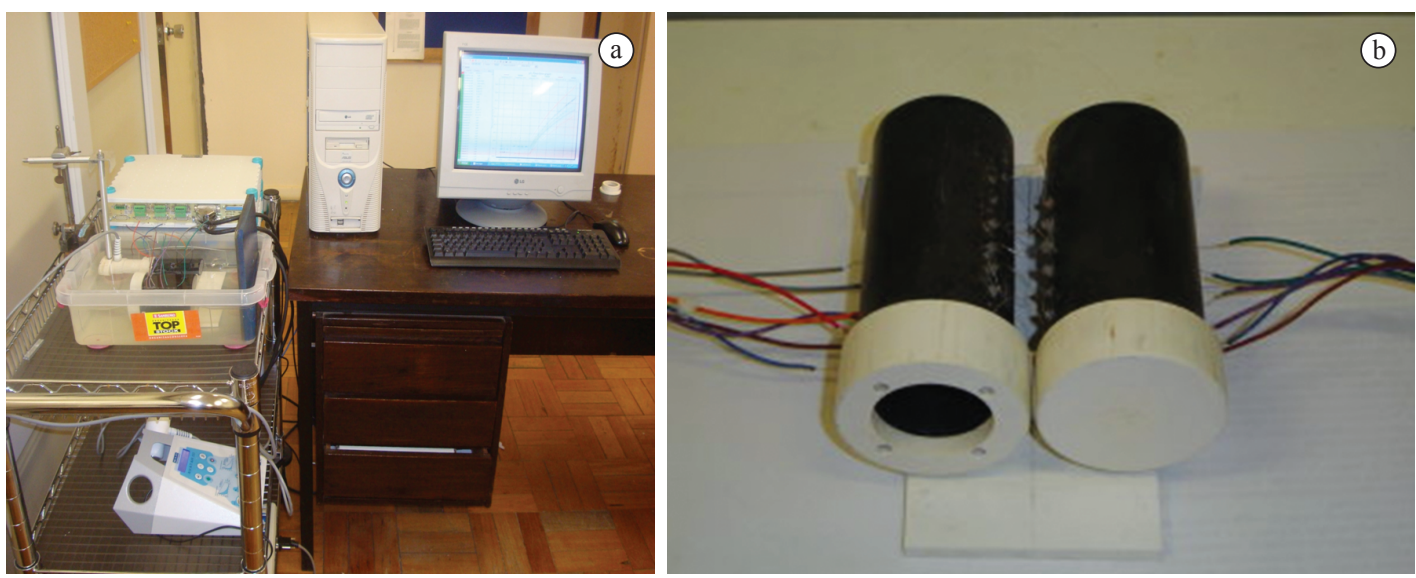

Figura 1. a) Montagem experimental para aquisição da temperatura no calorímetro: equipamento de ultrassom, Spider 8 , microcomputador e recipiente com água contendo o calorímetro diferencial, transdutor e placa absorvedora ultrassônica; b) Calorímetro diferencial construído: tubos de medição e de referência, termopares diferenciais e posicionador de PVC (tampa com orifício para permitir a passagem do campo ultrassônico e aquecimento do "phantom" no tubo de medição e tampa lisa no tubo de referência).

Figure 1. a) Experimental setup for aquisition of temperature in the calorimeter: ultrasound equipment, Spider 8, microcomputer and a container with water having the differential calorimeter, transducer and ultrassonic absorbing plate; b) Differential calorimeter: measurement and reference chambers, differential thermocouples and PVC positioner (cover with hole to allow the passage of the acoustic beam and phantom heating on the measurement chamber and smooth cover on the reference chamber). 
ultrassônico de fisioterapia (Avatar III, KLD), hidrofone (Needle Hydrophone, Precision Acoutics LTD) de diâmetro $0,5 \mathrm{~mm}$ e sensibilidade $1 \mathrm{MHz} 200,7 \mathrm{mV} / \mathrm{MPa}$ e $3 \mathrm{MHz}$ 185,5 mV/MPa e microcomputador. Para cada transdutor foram levantados cinco planos: um no eixo de propagação do feixe ultrassônico e quatro planos paralelos à face do transdutor (para o cálculo da ERA). Foram utilizados dois programas em Labview 8.2 (desenvolvidos no LUS): um para controlar o motor de passo e outro para calcular a ERA.

No mapeamento do campo acústico, utilizou-se um hidrofone (HPM05/3, Precision Acoustics LTD) em um plano ao longo do feixe acústico para definição da posição do foco (Zn) (Figura 3) e a quatro planos distantes da face do transdutor (Z1, Z2, Z3, Z4) (Figuras 4 e 5). A ERA estimada do transdutor de $1 \mathrm{MHz}$ foi de $3,31 \mathrm{~cm}^{2}$ (nominal de $5 \mathrm{~cm}^{2}$ ) e do transdutor de $3 \mathrm{MHz}$ foi de $3,58 \mathrm{~cm}^{2}$ (nominal de $5 \mathrm{~cm}^{2}$ ). Em seguida, a intensidade efetiva foi estimada a partir dos valores da potência acústica e da ERA, conforme a Tabela 1.

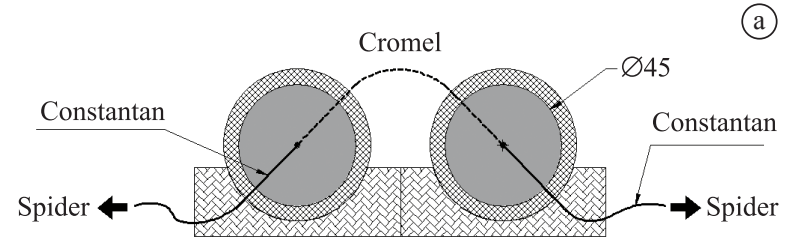

$\mathrm{O}$ aquecimento do phantom foi determinado a partir da variação de temperatura, ou seja, a diferença entre as temperaturas inicial e final do phantom $(\Delta \mathrm{T})$, correspondentes aos instantes pré e pós-irradiação ultrassônica.

As Tabelas 2 e 3 apresentam os resultados gerais dos experimentos, nas freqüências 1 e $3 \mathrm{MHz}$, respectivamente.

Também foram traçadas quatro curvas da média de aquecimento versus a profundidade, em um único plano, correspondentes ao padrão de aquecimento a cada intensidade nominal empregada $(0,5,1,0,1,5 \mathrm{e}$ $2,0 \mathrm{~W} \cdot \mathrm{cm}^{-2}$ ) ao longo do phantom (Figuras $6 \mathrm{e} 7$ ).

\section{Discussão}

No presente trabalho foi projetado e construído um calorímetro diferencial para se estudar o efeito da absorção ultrassônica.

Figura 2. a) Esquema do calorímetro diferencial composto de dois tubos cilíndricos: um de medição e outro de referência, ambos contendo o phantom e a junção de termopar. b) Corte longitudinal do calorímetro mostrando a posição dos 6 termopares no phantom, posicionador e transdutor. A distância entre os termopares foi de $10 \mathrm{~mm}$, e a distância entre a superfície do phantom e o primeiro termopar foi de $10 \mathrm{~mm}$. Figure 2. a) Differential calorimeter composed of two cylindrical chambers: measurement and reference, both containing the phantom and the thermocouple junction. b) Longitudinal view of the calorimeter presenting the position of the 6 thermocouples in the phantom, positioner and transducer. The distance between the thermocouples was $10 \mathrm{~mm}$, and the distance between the phantom surface and the first thermocouple was $10 \mathrm{~mm}$.

(a)

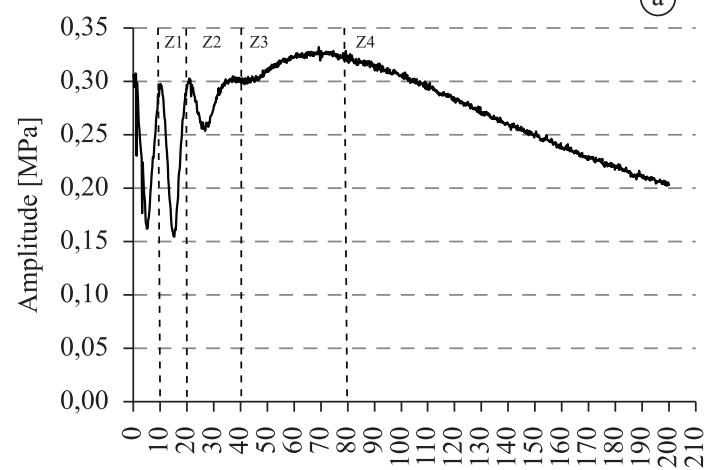

(b)

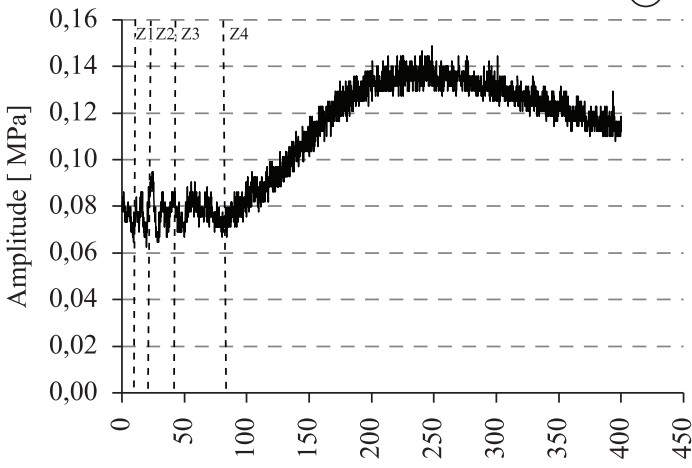

Figura 3. Varreduras ao longo do feixe ultrassônico no transdutor de $1 \mathrm{MHz}$, com posição do último pico (Zn) em $70 \mathrm{~mm}$ (a) e no transdutor de $3 \mathrm{MHz}$, com posição do último pico (Zn) em $240 \mathrm{~mm}$ (b). $Z n$ correspondente ao limite entre os campos próximo e distante. As linhas tracejadas Z1, Z2, Z3 e Z4 correspondem às posições onde foram realizadas as varreduras em 2D nestes planos.

Figure 3. Sweep along the ultrassonic beam on the 1-MHz transducer, with the latest peak (Zn) at $70 \mathrm{~mm}(\mathrm{a})$, and in the 3-MHz transducer, with the latest peak at $240 \mathrm{~mm}$ (b). Zn corresponds to the limit between the near and distant field. The dashed lines Z1, Z2, Z3 and Z4 correspond to positions where the sweeps were performed in these planes. 


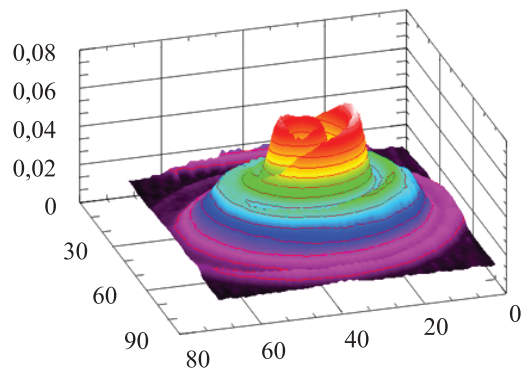

Z1

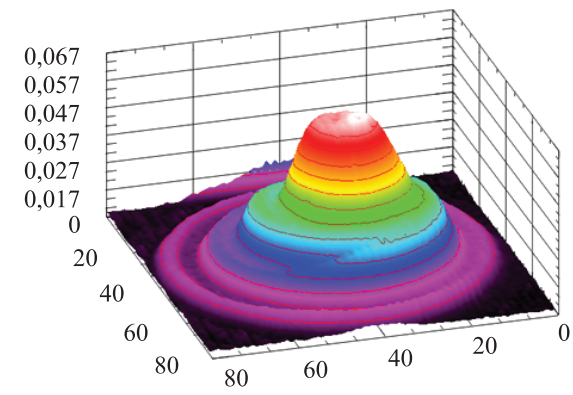

Z3

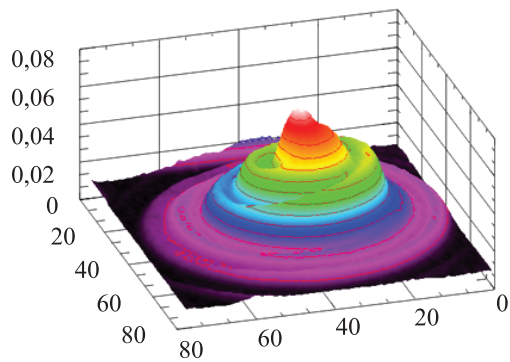

$\mathrm{Z} 2$

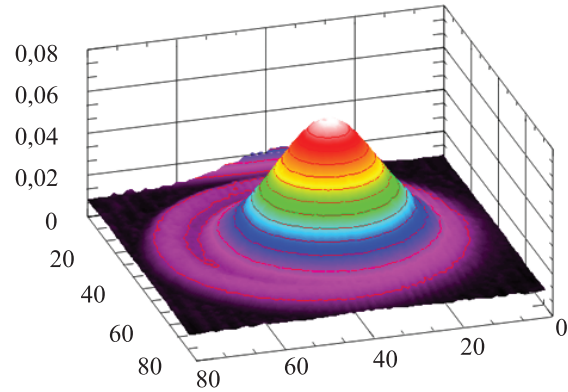

Z4

Figura 4. Distribuição da pressão acústica em planos paralelos à face do transdutor. Gráficos em 2D correspondentes aos planos Z1, Z2, Z3 e Z4 obtidos com a varredura do campo acústico do transdutor de $1 \mathrm{MHz}$ por hidrofone.

Figure 4. Graphics in 2D corresponding to the planes Z1, Z2, Z3 and Z4 obtained by the acoustic field sweep of the 1 MHz transducer by hydrophone.

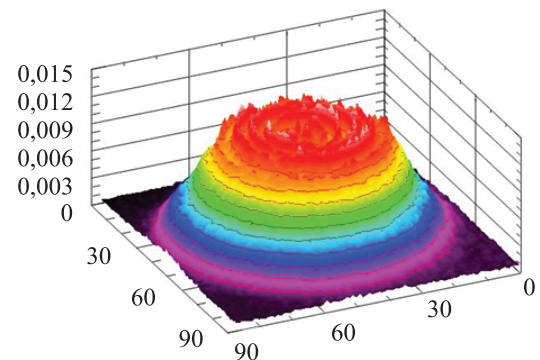

Z1

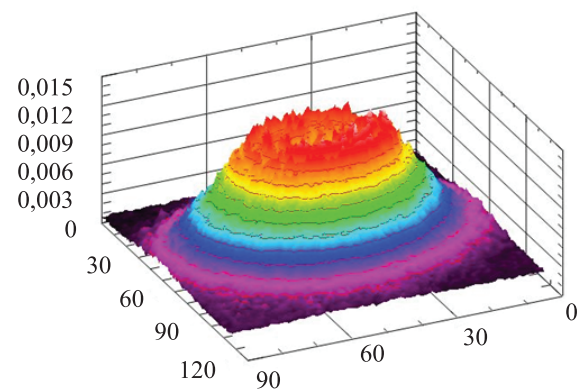

Z3

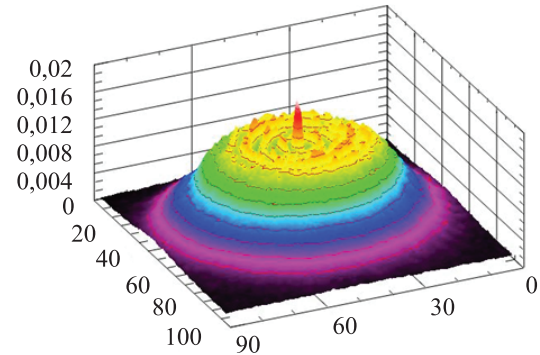

Z2

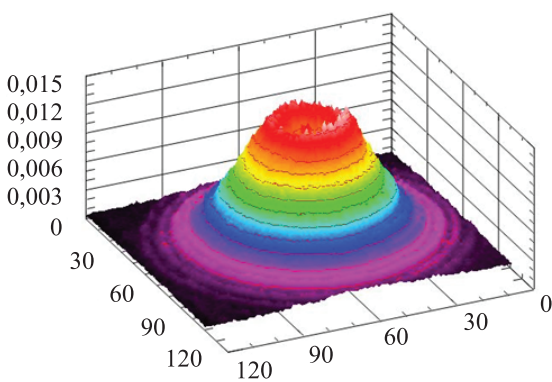

Z4

Figura 5. Distribuição da pressão acústica em planos paralelos à face do transdutor. Gráficos em $2 \mathrm{D}$ correspondentes aos planos Z1, Z2, Z3 e Z4 obtidos com a varredura do campo acústico do transdutor de $3 \mathrm{MHz}$ por hidrofone.

Figure 5. Graphics in 2D corresponding to the planes Z1, Z2, Z3 and Z4 obtained by the acoustic field sweep of the $3 \mathrm{MHz}$ transducer by hydrophone. 
Tabela 1. Comparação entre os valores de intensidade nominal e intensidade efetiva, proveniente da razão entre a potência e a ERA estimadas.

Table 1. Comparison between values of nominal and effective intensity, from the ratio between power and ERA estimated.

\begin{tabular}{ccc}
\hline $\begin{array}{c}\text { Intensidade } \\
\text { nominal } \\
{\left[\mathbf{W} \cdot \mathbf{c m}^{-2}\right]}\end{array}$ & $\begin{array}{c}\text { Intensidade } \\
\text { efetiva } \mathbf{~} \mathbf{~ M H z} \\
{\left[\mathbf{W} \cdot \mathbf{c m}^{-2}\right]}\end{array}$ & $\begin{array}{c}\text { Intensidade } \\
\text { efetiva } \mathbf{3 ~} \mathbf{~ M H z} \\
{\left[\mathbf{W} \cdot \mathbf{c m}^{-2}\right]}\end{array}$ \\
\hline 0,20 & 0,24 & 0,22 \\
0,40 & 0,55 & 0,48 \\
0,60 & 0,85 & 0,74 \\
0,80 & 1,16 & 1,03 \\
1,00 & 1,47 & 1,29 \\
1,20 & 1,79 & 1,58 \\
1,40 & 2,13 & 1,82 \\
1,60 & 2,44 & 2,12 \\
1,80 & 2,75 & 2,43 \\
2,00 & 3,08 & 2,72 \\
\hline
\end{tabular}

O tubo de alumínio utilizado no calorímetro foi dimensionado de forma que a secção transversal fosse cerca de 4,5 vezes a ERA dos transdutores empregados. Isto foi feito para minimizar o efeito de possíveis reflexões nas paredes do tubo. Outra preocupação foi utilizar termopares de diâmetro pequeno o suficiente para minimizar a interferência com o campo acústico $(0,24 \mathrm{~mm}$ de diâmetro). $\mathrm{O}$ alumínio foi o material selecionado para construção do calorímetro, por ser um bom condutor de calor, evitando assim, sobreaquecimento do phantom. Assim, o fluxo de calor dentro do phantom, deste para as paredes de alumínio e das paredes para a água se aproximava do mecanismo de condução de calor observado nos tecidos.

O modelo apresentado foi simplificado, não incluiu a simulação da perfusão sanguínea, o phantom

Tabela 2. Valores de média do aquecimento $\left[\Delta^{\circ} \mathrm{C}\right]$, desvio-padrão (DP) $\left[\Delta^{\circ} \mathrm{C}\right]$ e coeficiente de variação $(\mathrm{CV})$ gerado por transdutor de $1 \mathrm{MHz}$ em 6 profundidades do phantom.

Table 2. Data of heating average $\left[\Delta^{\circ} \mathrm{C}\right]$, standard deviation $(D P)\left[\Delta^{\circ} \mathrm{C}\right]$ and variation coefficient generated by $1-M H z$ transducer at 6 depths of the phantom.

\begin{tabular}{|c|c|c|c|c|c|c|c|}
\hline $1 \mathrm{MHz}$ & & $10 \mathrm{~mm}$ & $20 \mathrm{~mm}$ & $30 \mathrm{~mm}$ & $40 \mathrm{~mm}$ & $50 \mathrm{~mm}$ & $60 \mathrm{~mm}$ \\
\hline \multirow[t]{3}{*}{$0,5 \mathrm{~W} \cdot \mathrm{cm}^{-2}$} & Média & 1,69 & 1,50 & 1,31 & 1,34 & 1,08 & 1,19 \\
\hline & DP & 0,24 & 0,18 & 0,25 & 0,14 & 0,13 & 0,18 \\
\hline & CV (\%) & 15 & 12 & 19 & 10 & 12 & 15 \\
\hline \multirow[t]{3}{*}{$1,0 \mathrm{~W} \cdot \mathrm{cm}^{-2}$} & Média & 3,35 & 2,96 & 2,54 & 2,52 & 2,05 & 2,18 \\
\hline & DP & 0,47 & 0,31 & 0,47 & 0,15 & 0,14 & 0,27 \\
\hline & CV $(\%)$ & 14 & 10 & 18 & 6 & 7 & 12 \\
\hline \multirow[t]{3}{*}{$1,5 \mathrm{~W} \cdot \mathrm{cm}^{-2}$} & Média & 5,07 & 4,37 & 3,71 & 3,70 & 3,06 & 3,15 \\
\hline & DP & 0,72 & 0,49 & 0,68 & 0,26 & 0,18 & 0,33 \\
\hline & CV $(\%)$ & 14 & 11 & 18 & 7 & 6 & 10 \\
\hline \multirow[t]{3}{*}{$2,0 \mathrm{~W} \cdot \mathrm{cm}^{-2}$} & Média & 6,71 & 5,76 & 4,86 & 4,79 & 3,98 & 4,04 \\
\hline & DP & 0,96 & 0,64 & 0,89 & 0,30 & 0,21 & 0,37 \\
\hline & CV $(\%)$ & 14 & 11 & 18 & 6 & 5 & 9 \\
\hline
\end{tabular}

Tabela 3. Valores de média do aquecimento $\left[\Delta^{\circ} \mathrm{C}\right]$, desvio-padrão (DP) $\left[\Delta^{\circ} \mathrm{C}\right]$ e coeficiente de variação (CV) gerado por transdutor de $3 \mathrm{MHz}$ em 6 profundidades do phantom.

Table 3. Data of heating average $\left[\Delta^{\circ} \mathrm{C}\right]$, standard deviation $(D P)\left[\Delta^{\circ} \mathrm{C}\right]$ and variation coefficient generated by the 3 MHz transducer at 6 depths of the phantom.

\begin{tabular}{cccccccc}
\hline $\mathbf{3} \mathbf{~ M H z}$ & & $\mathbf{1 0} \mathbf{~ m m}$ & $\mathbf{2 0} \mathbf{~ m m}$ & $\mathbf{3 0} \mathbf{m m}$ & $\mathbf{4 0} \mathbf{~ m m}$ & $\mathbf{5 0} \mathbf{~ m m}$ & $\mathbf{6 0} \mathbf{m m}$ \\
\hline $0,5 \mathrm{~W} \cdot \mathrm{cm}^{-2}$ & Média & 2,97 & 2,35 & 1,87 & 1,46 & 1,21 & 1,00 \\
& DP & 0,35 & 0,27 & 0,20 & 0,17 & 0,13 & 0,20 \\
& $\mathbf{C V}(\mathbf{\%})$ & $\mathbf{1 2}$ & $\mathbf{1 1}$ & $\mathbf{1 1}$ & $\mathbf{1 1}$ & $\mathbf{1 1}$ & $\mathbf{2 0}$ \\
$1,0 \mathrm{~W} \cdot \mathrm{cm}^{-2}$ & Média & 6,04 & 4,83 & 3,81 & 2,97 & 2,47 & 2,01 \\
& DP & 0,61 & 0,42 & 0,35 & 0,17 & 0,17 & 0,28 \\
& CV(\%) & $\mathbf{1 0}$ & $\mathbf{9}$ & $\mathbf{9}$ & $\mathbf{6}$ & $\mathbf{7}$ & $\mathbf{1 4}$ \\
$1,5 \mathrm{~W} \cdot \mathrm{cm}^{-2}$ & Média & 9,22 & 7,37 & 5,77 & 4,46 & 3,66 & 2,96 \\
& DP & 0,92 & 0,70 & 0,57 & 0,27 & 0,24 & 0,41 \\
& CV(\%) & $\mathbf{1 0}$ & $\mathbf{9}$ & $\mathbf{1 0}$ & $\mathbf{6}$ & $\mathbf{6}$ & $\mathbf{1 4}$ \\
$2,0 \mathrm{~W} \cdot \mathrm{cm}^{-2}$ & Média & 12,56 & 10,03 & 7,77 & 5,99 & 4,92 & 3,96 \\
& DP & 1,22 & 0,94 & 0,76 & 0,52 & 0,41 & 0,61 \\
\hline
\end{tabular}


empregado foi homogêneo (camada única) e a forma de aplicação do transdutor foi estática (que difere da prática clínica). Sendo assim, não é possível extrapolar diretamente tais resultados para casos in vivo, porém permitem que se tenha uma ideia de como o aquecimento do volume ocorre.

A ERA estimada de acordo com a norma NBR 1689 (1998) foi menor do que a ERA nominal a 1 e 3 MHz.
A $1 \mathrm{MHz}$, notou-se um declínio da taxa de aquecimento ao longo da profundidade em todas as intensidades, embora este seja mais acentuado em intensidades nominais mais elevadas $\left(1,5\right.$ e $\left.2,0 \mathrm{~W} \cdot \mathrm{cm}^{-2}\right)$. Para cada intensidade nominal, foram observados dois picos de temperatura no calorímetro: a $10 \mathrm{~mm}$ e a $60 \mathrm{~mm}$. Estes picos corresponderam às distâncias do campo acústico de $20 \mathrm{~mm}$ e $70 \mathrm{~mm}$, respectivamente.

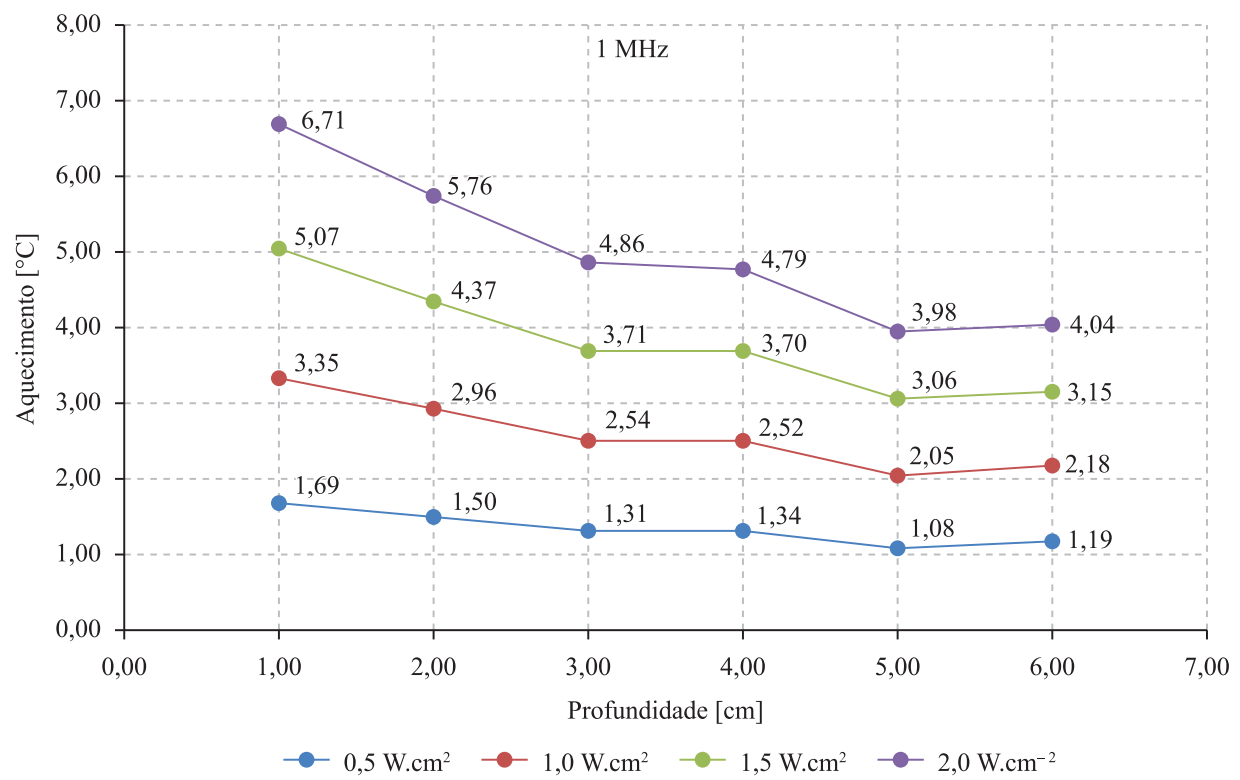

Figura 6. Curvas da média (de 10 valores) do aquecimento versus profundidade $(1-6 \mathrm{~cm})$ do phantom a $1 \mathrm{MHz}$, nas intensidades 0,$5 ; 1,0$; 1,5 e $2,0 \mathrm{~W} \cdot \mathrm{cm}^{-2}$.

Figure 6. Average curves (10 values) of heating versus depth (1-6 cm) of the phantom at $1 \mathrm{MHz}$, at intensities 0,5; 1,0; 1,5 e 2,0 W. $\mathrm{cm}^{-2}$.

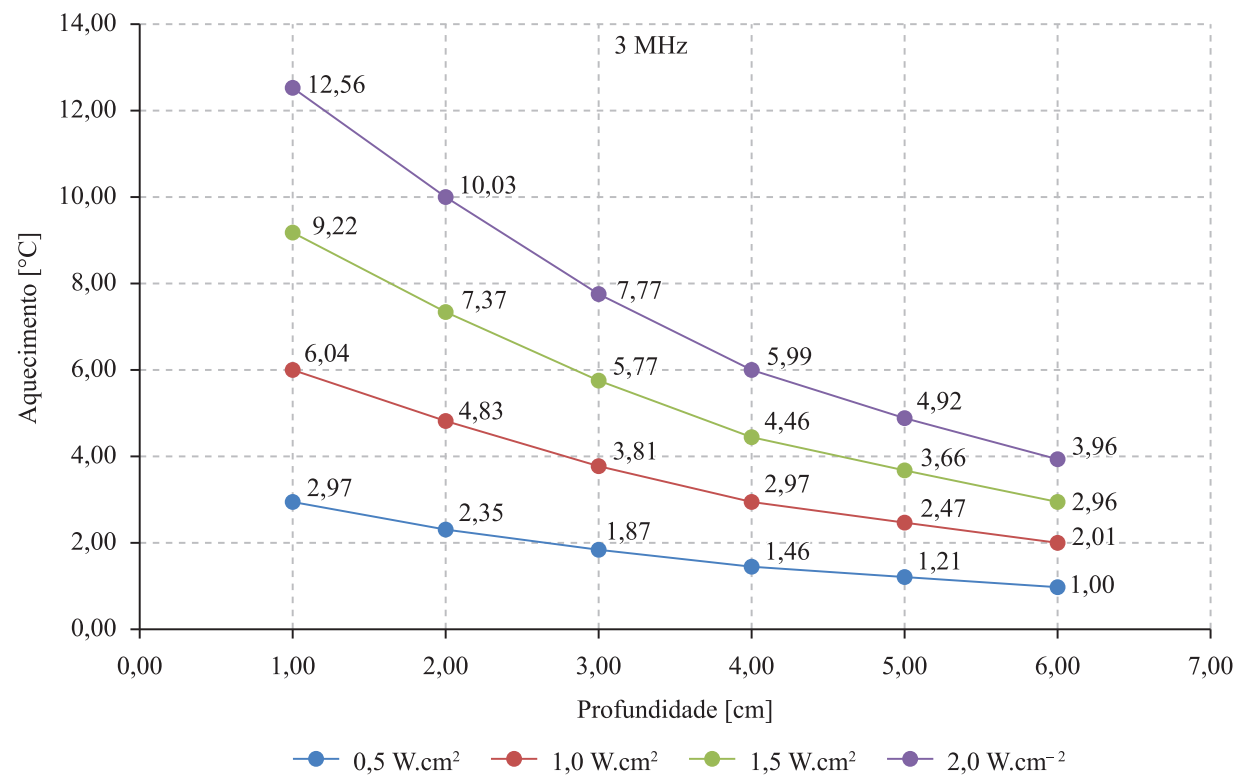

Figura 7. Curvas da média (de 10 valores) do aquecimento versus profundidade (1-6 cm) do phantom) a $3 \mathrm{MHz}$, nas intensidades 0,5 ; 1,0 ; 1,5 e $2,0 \mathrm{~W} \cdot \mathrm{cm}^{-2}$.

Figure 7. Average curves (10 values) of heating versus depth (1-6 cm) of the phantom at $3 \mathrm{MHz}$, at intensities 0,$5 ; 1,0 ; 1,5$ e $2,0 \mathrm{~W} \cdot \mathrm{cm}^{-2}$. 
O pico observado a $10 \mathrm{~mm}$ correspondeu a um pico de intensidade no campo próximo, que se situou na região mais superficial do phantom. $\mathrm{O}$ segundo pico de temperatura ocorreu para o termopar a $60 \mathrm{~mm}$. Observou-se que este aqueceu mais do que o a $50 \mathrm{~mm}$, em todas as intensidades nominais. Uma possível explicação foi sua proximidade com o foco natural do feixe ultrassônico (que está a $70 \mathrm{~mm}$ ).

A $3 \mathrm{MHz}$ foram observados picos de temperatura superficialmente, principalmente nos termopares a $10 \mathrm{~mm}$ e $20 \mathrm{~mm}$ de profundidade, e declínio da taxa de aquecimento com a profundidade, em todas as intensidades. A variação da pressão ultrassônica na varredura ao longo do feixe ultrassônico do transdutor de $3 \mathrm{MHz}$, se mostrou menor $(0,06-0,16 \mathrm{MPa})$ do que a amplitude em $1 \mathrm{MHz}(0,15-0,35 \mathrm{MPa})$. Além disso, a intensidade efetiva calculada do transdutor de $1 \mathrm{MHz}$ foi maior do que a de $3 \mathrm{MHz}$. Apesar disso, o aquecimento a $3 \mathrm{MHz}$ foi maior do que a $1 \mathrm{MHz}$ nas profundidades $10-50 \mathrm{~mm}$, e menor na profundidade $60 \mathrm{~mm}$, em todas as intensidades nominais. Isto pode ser atribuído à absorção ser maior na freqüência de $3 \mathrm{MHz}$, o que leva à maior produção de calor nas regiões superficiais.

Também pode-se observar que a região do "phantom" que mais aqueceu foi a que corresponde à profundidade de $10 \mathrm{~mm}$ em ambas as frequiências. Esta resposta também foi relatada por Kadri et al. (2007). Apesar da freqüência de $1 \mathrm{MHz}$ ser rotineiramente indicada para tratamento de lesões teciduais profundas e a freqüência de $3 \mathrm{MHz}$ para lesões teciduais superficiais (Prentice, 2004), o aquecimento superficial ocorreu em ambas as freqüências, embora com magnitudes diferentes: na mesma intensidade nominal, a $3 \mathrm{MHz}$, o aquecimento entre $10-30 \mathrm{~mm}$ foi mais acentuado. A partir desta profundidade (40-60 $\mathrm{mm})$, houve uma pequena diferença de aquecimento entre as freqüências.

A partir dos dados experimentais deste trabalho, a radiação emitida pelo transdutor de $3 \mathrm{MHz}$ (dito de ação superficial) proporcionou maior aquecimento até $50 \mathrm{~mm}$ de profundidade do que o de $1 \mathrm{MHz}$ (dito de ação profunda) quando aplicado em um phantom com propriedades acústicas equivalentes às da gordura, mesmo com a intensidade efetiva um pouco menor do que a $1 \mathrm{MHz}$. Ou seja, é razoável se supor que, se o transdutor de $3 \mathrm{MHz}$ se encontrasse com intensidade semelhante à gerada pelo transdutor de $1 \mathrm{MHz}$, o aquecimento promovido na frequência de $3 \mathrm{MHz}$ continuaria sendo mais elevado. Supondo dependência linear do coeficiente de atenuação com a frequência (Wells, 1977), a $3 \mathrm{MHz}$, o coeficiente de atenuação seria $0,66 \mathrm{~dB} \cdot \mathrm{cm}^{-1}$, o que ainda é bem abaixo do coeficiente de atenuação para o músculo $\left(1,8 \mathrm{~dB} \cdot \mathrm{cm}^{-1}\right)$. Sendo assim, o feixe a $3 \mathrm{MHz}$ no phantom aqui utilizado tende a penetrar mais, portanto, aquecendo regiões mais profundas. Nos casos reais a terapia não visa o aquecimento da gordura, e sim, de tendões, músculos, etc., que possuem coeficientes de atenuação mais altos.

Os resultados deste trabalho evidenciaram a capacidade do UST (Ultrassom terapêutico) em promover aquecimento em níveis terapêuticos, nos tecidos. Por outro lado, foram verificados valores extremos de aquecimento na intensidade nominal de $2,0 \mathrm{~W} \cdot \mathrm{cm}^{-2}$, no termopar a $10 \mathrm{~mm}$, tanto em $1 \mathrm{MHz}$ $\left(6,7 \pm 1,0{ }^{\circ} \mathrm{C}\right)$ quanto em $3 \mathrm{MHz}\left(12,6 \pm 1,2{ }^{\circ} \mathrm{C}\right)$, temperaturas potencialmente lesivas.

\section{Conclusão}

Este trabalho apresentou o projeto e a construção de um calorímetro diferencial, composto por termopares imersos em material mimetizador de propriedades acústicas e térmicas da gordura. Tal calorímetro tem potencial para ser usado na caracterização do feixe de transdutores de ultrassom aplicados à Fisioterapia, em especial, na identificação dos principais máximos locais de temperatura (pontos quentes) que ocorrem ao longo do eixo principal do feixe. Serve, também, de base para se estudar doses adequadas (combinação de intensidade e tempo de aplicação) para que a temperatura se mantenha em níveis terapêuticos.

\section{Agradecimentos}

À CAPES, CNPq e FAPERJ pelo apoio financeiro.

\section{Referências}

Alvarenga AV, Machado JC, Pereira WCA. Implementação do protocolo para a obtenção de parâmetros do feixe acústico de transdutores ultra-sônicos usados em medicina. Revista Brasileira de Engenharia Biomédica. 2001; 17(3):151-63.

Associação Brasileira de Normas Técnicas - ABNT. NBR/IEC 1689: 1998. Ultra-som - Sistemas de Fisioterapia - Prescrições para desempenho e métodos de medição na faixa de freqüências de $0,5 \mathrm{MHz}$ a $5 \mathrm{MHz}$. Brasil: ABNT; 1998.

Basto RLQ. Construção de um phantom sintético com propriedades termofísicas e ultrassônicas similares às dos tecidos biológicos humano em 1,0 e 2,8 MHz [dissertação]. Rio de Janeiro: Universidade Federal do Rio de Janeiro; 2006.

Cameron MH. Ultrassom. In: Cameron MH. Agentes físicos na reabilitação. 3th ed. São Paulo: Elsevier; 2009. p. 177-206.

Delchar TA, Melvin RJ. A calorimeter for ultrasound total power measurements. Measurement Science and Technology. 1994; 5(12):1533-7. http://dx.doi. org/10.1088/0957-0233/5/12/015 
Draper DO, Ricard MD. Rate of temperature decay in human muscle following $3 \mathrm{MHz}$ ultrasound: the stretching window revealed. Journal of Athletic Training. 1995; 30(4):304-7. PMCid:1317998.

Fish P. Physics and instrumentation of diagnostic medical ultrasound. 2nd ed. New York: John Wiley \& Sons; 1990.

Furtado CS. Método calorimétrico para medição de campo térmico gerado por ultra-som empregado em Fisioterapia [dissertação]. Rio de Janeiro: Universidade Federal do Rio de Janeiro; 2005.

Guirro ECO, Guirro RR. Ultra-som. In: Guirro ECO, Guirro RR. Fisioterapia dermato-funcional: Fundamentos, recursos, patologias. 3. ed. São Paulo: Manole; 2004. p. 175-208.

Hayes BT, Merrick MA, Sandrey MA, Cordova ML. Three-MHz ultrasound heats deeper into the tissues than originally theorized. Journal of Athletic Training. 2004; 39(3):230-4. PMCid:522144.

Ishikawa NM. Avaliação de equipamentos de ultra-som para Fisioterapia segundo a norma NBR IEC 1689 da Associação Brasileira de Normas Técnicas [dissertação]. Rio de Janeiro: Universidade Federal do Rio de Janeiro; 2000.

Johns LD, Straub SJ, Howard SM. Variability in effective radiating area and output power of new ultrasound transducers at $3 \mathrm{MHz}$. Journal of Athletic Training. 2007; 42(1):22-8. PMCid:1896073.
Kadri NA, Ahamad AR, Abdul-Latip EN, Azlan CA, Raha MG, Mohd-Nasir NF. Temperature modeling of therapeutic ultrasound: a preliminary finding. IFMBE Proceedings. 2007; 15(15):594-7. http://dx.doi. org/10.1007/978-3-540-68017-8_149

Merrick MA, Bernard KD, Devor ST, Williams MJ. Identical 3-MHz ultrasound treatments with different devices produce different intramuscular temperatures. Journal of Orthopaedic and Sports Physical Therapy. 2003; 33(7):379-85. PMid:12918863.

Prentice WE. Ultra-som terapêutico. In: Prentice WE. Modalidades terapêuticas para fisioterapeutas. 2. ed. Porto Alegre: Artmed; 2004. p. 245-82.

Ramos CBZ. Diseño de un sistema para la medición de potencia US por medio de calorimetría para transductores de terapia [dissertação]. México: Centro de Investigación y de Estúdios Avanzados del Instituto Politécnico Nacional; 2004.

Straub SJ, Johns LD, Howard SM. Variability in effective radiating area at $1 \mathrm{MHz}$ affects ultrasound treatment intensity. Physical Therapy. 2008; 88(1):51-7. PMid:17940107.

Wells PNT. Velocity, absorption and attenuation in biological materials. In: Wells PNT. Biomedical Ultrasonics. New York: Academic Press; 1977. p. 110-44.

\footnotetext{
Autores

Thaís Pionório Omena*

Faculdade de Medicina, Universidade Federal do Rio de Janeiro - UFRJ, Rio de Janeiro, RJ, Brasil. Laboratório de Ultrassom, Programa de Engenharia Biomédica, Instituto Alberto Luiz Coimbra de Pós-Graduação e Pesquisa de Engenharia - COPPE, Universidade Federal do Rio de Janeiro - UFRJ, Av. Horácio Macedo, 2030, Bloco H, Sala 327, CEP 21941-914, Rio de Janeiro, RJ, Brasil.

Centro de Tecnologia, Instituto Alberto Luiz Coimbra de Pós-Graduação e Pesquisa de Engenharia - COPPE, Universidade Federal do Rio de Janeiro - UFRJ, Cidade Universitária, CP 68510, CEP 21941-972, Rio de Janeiro, RJ, Brasil.
}

Rejane Medeiros Costa, Wagner Coelho de Albuquerque Pereira, Marco Antônio von Krüger

Programa de Engenharia Biomédica, Instituto Alberto Luiz Coimbra de Pós-Graduação e Pesquisa de Engenharia - COPPE, Universidade Federal do Rio de Janeiro - UFRJ, Rio de Janeiro, RJ, Brasil. 